\title{
Somatotipo y Estado Nutricional de 10 a 14 Años de Edad en una Muestra de Mapuches de la IX Región, Temuco-Chile
}

\author{
Somatotype and Nutritional Status from 10 to 14 Years of Age \\ in a Sample of Mapuche Subjects in the IX Region, Temuco-Chile \\ "Cristián Martínez; * Héctor Silva; **Erika Collipal; *Vanessa Carrasco; \\ ***** Manuel Rodríguez; ${ }^{* * * *}$ Rodrigo Vargas; ${ }^{* * * *}$ Patricio Gatica \& ${ }^{* * * * *}$ Tamara Silva
}

\begin{abstract}
MARTÍNEZ, C.; SILVA, H.; COLLIPAL, E.; CARRASCO, V; RODRÍGUEZ, M.; VARGAS, R.; GATICA, P. \& SILVA, T. Somatotipo y estado nutricional de 10 a 14 años de edad en una muestra de mapuches de la IX Región, Temuco-Chile. Int. J. Morphol., 30(1):241-246, 2012.

RESUMEN: El grupo étnico mapuche tiene una amplia distribución en la Novena Región, el proceso migratorio propio del desarrollo urbano, han hecho que parte de la etnia mapuche se traslade a la ciudad de Temuco, modificando sus estilos de vida. El estudio del Índice de Masa Corporal (IMC), es el parámetro recomendado por la OMS (Organización Mundial de la Salud), para conocer el estado nutricional de acuerdo a la norma técnica nutricional vigente en Chile. El somatotipo es utilizado para estimar la forma corporal y su composición. Este estudio describe la realidad nutricional y el somatotipo de una muestra de mapuches de los colegios municipalizados de la ciudad de Temuco. Se evaluaron 292 adolescentes mapuches de ambos sexos, 154 hombres (52,7\%) y 138 mujeres (47,3\%), entre 10 y 14 años de edad. Para obtener el somatotipo se utilizó el método antropométrico descrito por Heath \& Carter mientras que la evaluación y clasificación del estado nutricional de acuerdo a las normas técnica nutricional. Los hombres presentaron un somatotipo mesoendomórficos (4.9/4.4). Las mujeres son endomesomórfico (5.4/4.3). El estado nutricional de la muestra tiene los siguientes resultados en el caso de los varones el 11,7\% fueron obesos y el 20,1\% presentaron sobrepeso. En las damas el 9,5\% fueron obesas y un 27,5 $\%$ presentaron sobrepeso. Al comparar por sexo se encontraron diferencias significativas en la Endomorfía a favor de las mujeres ( $\mathrm{p}$ $=0.00)$ y en los varones la Mesomorfía, con un $(\mathrm{p}=0.00)$. No así en la Ectomorfía que no presentó diferencias. No obstante, no se encontraron diferencias al comparar los componentes del somatotipo entre las edades de la muestra. El ambiente Obesigénico donde destaca la modificación del estilo de vida, la alimentación, el efecto del sedentarismo, está afectando de igual forma a la población mapuche que al resto de la población escolar al ser comparados con muestras de estudiantes de establecimientos municipalizados.
\end{abstract}

PALABRAS CLAVE: Mapuches; Somatotipo; Índice de masa corporal; Estado Nutricional.

\section{INTRODUCCIÓN}

Existen ocho grupos étnicos reconocidos en Chile, de acuerdo a la información del censo 2002 (INE, 2003). Estos equivalen al 4,6\% de la población nacional, siendo los mapuches el grupo mayoritario que representa el 87,3\% del total de las etnias presentes en el país. El grupo étnico mapuche tiene una amplia distribución de asentamiento, bajo el sistema reduccional en la IX Región de Chile (del Sol \& Hunter, 2004) y corresponde al 23,4\% del total de la población de la IX Región según el Instituto Nacional de Estadísticas (INE). La distribución de la población indígena en el país según áreas urbanas o rurales, muestra una migración de lo rural a lo urbano, un 79,63\% declaró vivir en ciudades y solo el 20,37\% restante habita en sectores rurales (Valenzuela, 1995).

La Organización Mundial para la Salud (OMS, 2009), define la obesidad como una enfermedad crónica y multifactorial, con numerosas complicaciones; resultado de la unión de factores biológicos, genéticos y ambientales. Los factores más estudiados de la obesidad están relacionados con los estilos de vida, actividad física, nivel socioeconómico, el género, y la edad, por lo tanto es compleja y dinámica (Peña \& Bacallao, 2006). En nuestro país, el Ministerio de Salud (2003) propuso utilizar el Índice de

* Departamento de Educación Física, Universidad de La Frontera. Temuco-Chile.

** Departamento de Ciencias Básicas, Universidad de La Frontera. Temuco-Chile.

*** Departamento de Didáctica de la Lengua y la Literatura Ciencias Sociales y la Educación Física Deportiva. Universidad de Almería. Almería- España.

**** Departamento de Ciencias de la Actividad Física. Universidad Católica del Maule. Talca-Chile.

******* Ayudante de Investigación de la carrera de Biotecnología. Universidad de La Frontera. Temuco-Chile. 
Masa Corporal (IMC) por edad como criterio de evaluación nutricional en escolares y adolescentes.

Un estudio reciente efectuado en una muestra nacional de escolares de $8^{\circ}$ básico edades entre 13-15 años encontró que el 40,2\% de ellos presentaban sobrepeso u obesidad (MINEDUC, 2010). Lo más preocupante es que la condición física global de esta misma muestra se encontró deficiente en el 90,8\% de los casos. Esta información no existe para otros grupos de edad escolar. Coincidentemente, datos existentes en adultos provenientes de 5 Regiones del país incluida la IX Región, muestran que la situación de mala condición física estuvo presente en el 84,5\% de los casos (Díaz et al., 2008). En la encuesta de salud del año 2010, la prevalencia de exceso de peso en la población adulta es del $64,5 \%$, la cual corresponde al $39,32 \%$ de sobrepeso y un $25,13 \%$ de obesidad (Ministerio de Salud, 2010).

La obesidad y el sobrepeso, unido a las enfermedades derivadas de ellas, se han convertido en uno de los mayores azotes en la sociedad a nivel mundial, con carácter epidemiológico. Convirtiéndose en uno de los mayores factores de riesgo cardiovascular para una buena parte de las enfermedades crónicas, siendo responsable de alrededor del $80 \%$ de obesidad tipo II en adultos, del $35 \%$ en casos de cardiopatía isquémica y del 55\% de casos de hipertensión arterial en adultos de países europeos (Manolles et al., 2008). Entre otros riesgos, el sobrepeso en adolescentes incrementa el riesgo de padecer enfermedad coronaria arterial y síndrome metabólico en edad adulta (Alegría et al., 2005; Bibbins et al., 2007).

La antropometría tiene como finalidad conocer las características morfológicas de una población. Utilizando la técnica del somatotipo, que describe la estructura corporal en tres componentes, Endomórfico, Mesomórficos y Ectomórfico. El concepto vigente del Somatotipo fue propuesto por Heath y Carter, quienes se apoyaron en los conceptos teóricos de Sheldon del año 1940 (Carter \& Heath, 1990). El análisis de los pliegues cutáneos permite estimar el contenido de masa grasa y masa libre de grasa en los diferentes individuos y consecuentemente, inferir los requerimientos nutricionales (Toro \& Almagià, 1989,1994).

Los estudios antropométricos permiten estimar la composición corporal, estudiar la morfología, dimensiones y proporcionalidad en relación al rendimiento deportivo, nutrición y crecimiento (Carter \& Heath; Cheng-Ye \& Ohsawa, 1996; Sánchez-Muñoz et al., 2007; Álvero et al., 2009).

El análisis del somatotipo ha sido realizado en poblaciones normales y deportistas de diferentes edades, sexo y niveles socioeconómicos para conocer las características biotipológicas de los diferentes grupos étnicos (Carter et al.,
1997; Katzmarzyk et al., 1998, 1999; Rahmawati et al., 2007; Ventrella et al., 2008). En los estudios de crecimiento el somatotipo permite monitorear patrones de crecimiento y caracterizar los cambios morfológicos y entender mejor las variaciones en el físico adulto (Norton \& Olds, 2000; Malina et al., 2004).

El somatotipo son datos de gran valor para caracterizar los cambios físicos durante el crecimiento y el envejecimiento. Permite comparar la forma relativa de hombres y mujeres. El índice de masa corporal evalúa el estado nutricional tanto en condiciones de salud, como de enfermedad (Toro \& Almagià, 1989, 1994; Silva et al., 2008; Manolles et al.; Alvero et al.). Valores específicos del somatotipo y de sus componentes han sido correlacionados en diferentes patologías, cáncer de mama, cardiopatías, escoliosis, obesidad y osteoporosis (Magnusson et al., 1998; Saitoglu et al., 2007). Además, en el deporte permite comparar entre especialidades, sexo y señalar las tendencias relacionas al somatotipo por disciplinas deportivas y por edad (Valkov et al., 1996; Carter \& Ackland, 1998) y realizar la búsqueda de talentos deportivos y modificar los componentes, mejorando el rendimiento según el deporte que se practique (Toro et al., 1983; Almagià et al.,1996).

En Chile, se han encontrado una gran variedad de somatotipos en la población escolar, que presentan características endomesomórficos y mesoendomórficos, observándose un predominio del componente endomesomórfico en damas, en los estudios relacionados con adolescentes (Silva et al., 2003, 2005, 2008; Martínez et al., 2008).

Han sido varios los estudios que se han realizado en los últimos años con la etnia mapuche analizando diferentes aspectos morfológicos, nutricionales y posturales, encontrándose variaciones morfológicas atribuibles a la raza (del Sol \& Henríquez, 1987; del Sol et al., 1990; del Sol \& Olave, 1996; Henríquez et al., 2003), deficiencias nutricionales (Ferrari et al., 2004). También se han realizado análisis posturales, faciales, cefálicos (del Sol \& Hunter; del Sol, 2005, 2006). No obstante, dichos sujetos no han sido evaluados en los componentes de su somatotipo e índice nutricional. Por tanto el objetivo de este estudio es analizar el somatotipo y determinar su estado nutricional en una muestra de estudiantes de la etnia mapuche de 10 a 14 años de la IX Región.

\section{MATERIAL Y MÉTODO}

Para el estudio del somatotipo y del IMC, se evaluaron 292 mapuches, 154 hombres $(52,7 \%)$ y 138 mujeres $(47,3 \%)$ cuyas edades fluctuaron entre los 10 y 14 años, provenientes de de colegios municipalizados de la ciudad de 
Temuco, IX Región, Chile. Se considero en la muestra solo a los sujetos que presentaban ambos apellidos mapuches.

Los sujetos fueron evaluados en sus colegios. Para la estimación del Somatotipo, se aplico el método de HeathCarter, los datos fueron registrados en una ficha antropométrica donde se registró el peso, la talla. Con antropómetro y cinta antropométrica de metal marca Rosscraft se evaluó los perímetros de brazo, pantorrilla, y los diámetros humeral y femoral. Con un adipómetro de marca Harpenden, se midieron los siguientes pliegues tríceps, subescapular, supraespinal, pantorrilla. Para la medición del peso corporal, se empleó una balanza digital marca Tanita modelo UM 2204 precisión de 0,2 kilos con una capacidad máxima de $136 \mathrm{~kg}$ la talla fue medida usando un estadíometro de pared, graduado en mm. La evaluación se hizo empleando técnicas y estándares descritos por ISAK (2001).

La escala de rating y características del somatotipo fue descrita por Carter \& Heath, el somatotipo se puede caracterizar por medio de valores que son cualitativos y cuantitativos, y van en rangos 1 a 21/2, 3 a 5, 51/2 a 7, 71/2 a 81/ 2, la Endomorfía se denomina como bajo, moderado, alta adiposidad y extremadamente alta adiposidad. Para la Mesomorfía es bajo desarrollo, moderado desarrollo, alto desarrollo, desarrollo músculo esquelético relativo extremadamente alto. Para la Ectomorfía, gran volumen por unidad de altura, linealidad relativa moderada, linealidad relativa elevada, linealidad relativa extremadamente alta.

El IMC se calculó a través de la formula Peso $(\mathrm{kg}) /$ Talla $(\mathrm{m})^{2}$ usando la clasificación propuesta en la norma técnica de evaluación nutricional de niños y niñas de 6 a 18 años del Ministerio de Salud de Chile (2004). Según este indicador se clasificó como sigue: bajo peso menor al percentil (p) 10, normal entre p10-p85, sobrepeso entre p85p95.

Para el procesamiento y análisis de los datos se utilizó el programa estadístico SPSS versión 17.0 analizando estadísticos descriptivos y estableciendo las posibles dife- rencias significativas entre las variables por género y entre los grupos de las diferentes edades, mediante las pruebas estadísticas, U de Mann Whitney y Kruskal- Wallis. Se empleó un error de $\mathrm{a}=0,05$ para establecer el nivel de significancia de las comparaciones antes citadas.

\section{RESULTADOS}

La clasificación del somatotipo e índice de masa corporal en adolecentes mapuches de ambos sexos según edad en Temuco-Chile y los componentes del somatotipo en la misma muestra, pueden ser observados en las Tablas I y II En la Tabla III se expresan los valores y porcentajes del estado nutricional de ambos sexos.

Tabla II. Promedio y DE de los componentes del somatotipo divididos por sexo en una muestra de adolescentes Mapuches de la ciudad de Temuco- Chile.

\begin{tabular}{lcccc}
\hline & \multicolumn{2}{c}{ Hombres } & \multicolumn{2}{c}{ Mujeres } \\
\hline & Promedio & DE & Promedio & DE \\
\hline Endomorfía & 4,4 & 1,1 & $* 5,4$ & 1,2 \\
Mesomorfía & $* 4,9$ & 1,8 & 4,3 & 1,6 \\
Ectomorfía & 2,3 & 1,3 & 2,1 & 1,4 \\
\hline
\end{tabular}

$\mathrm{n}=292 . *$ Existen diferencias significativas $\mathrm{p}=0,00$.

Tabla III. Diagnostico nutricional de una muestra de adolescentes Mapuches de ambos sexos de la ciudad de Temuco- Chile. $\mathrm{n}=292$.

\begin{tabular}{lcccccc}
\hline & \multicolumn{2}{c}{ Hombres } & \multicolumn{2}{c}{ Mujeres } & \multicolumn{2}{c}{ Total } \\
\cline { 2 - 7 } & $\mathbf{n}$ & $\boldsymbol{\%}$ & $\mathbf{n}$ & $\boldsymbol{\%}$ & $\mathbf{n}$ & $\boldsymbol{\%}$ \\
\cline { 2 - 7 } Obeso & 18 & 11,7 & 13 & 9,5 & 31 & 10,61 \\
Sobrepeso & 31 & 20,1 & 38 & 27,5 & 69 & 23,63 \\
Normal & 95 & 61,7 & 73 & 52,9 & 168 & 57,53 \\
Bajo peso & 10 & 6,5 & 14 & 10,1 & 24 & 8,23 \\
\hline
\end{tabular}

Tabla I. Clasificación del somatotipo en una muestra de adolescentes Mapuches divididos por sexo y edad de Temuco - Chile.

\begin{tabular}{lcccccccc}
\hline Edad & \multicolumn{2}{c}{$\mathbf{n}$} & \multicolumn{2}{c}{ Endomorfía } & \multicolumn{2}{c}{ Mesomorfía } & \multicolumn{2}{c}{ Ectomorfía } \\
\cline { 2 - 8 } & Hombres & Mujeres & Hombres & Mujeres & Hombres & Mujeres & Hombres & Mujeres \\
\hline 10 & 9 & 6 & 4,8 & 4,2 & 4,7 & 3,6 & 2,3 & 2,9 \\
11 & 43 & 27 & 4,2 & 5,4 & 4,8 & 4,2 & 2,2 & 2,3 \\
12 & 40 & 35 & 4,7 & 5,2 & 5,1 & 4,3 & 2,1 & 2,2 \\
13 & 46 & 41 & 4,7 & 5,6 & 4,9 & 4,4 & 2,4 & 1,9 \\
14 & 16 & 29 & 3,3 & 5,7 & 4,8 & 4,4 & 2,8 & 1,7 \\
\hline
\end{tabular}

$\mathrm{n}=292 *$ Existen diferencias significativas $\mathrm{p}=0,00$ 


\section{DISCUSIÓN}

Los resultados encontrados permiten evaluar y comparar que una muestra de la etnia mapuche se comporta de modo similar a las características de estado nutricional y somatotipo de la población general de escolares. Los cuales nos indican que se mantiene la tendencia, del estado nutricional y somatotipo en los estudios anteriores realizados en Chile, en que la población femenina presenta un somatotipo endomesomórfico y los varones meso-endomórficos de acuerdo a lo encontrado para población escolar municipalizada de la IX región por (Silva et al., 2005, 2008; Martínez et al.).

Este somatotipo de la población femenina es indicativo de un mayor contenido de tejido adiposo sobre el muscular y la linealidad, condición que se presenta en las edades de 10 a 14 años en las Damas y solo en los 10 años en los varones, en las otras edades de los hombres el somatotipo predominante es mesoendomórficos.

Al comparar por sexo, se encuentran diferencias significativas en cuanto a la endomorfía a favor de las mujeres $(p=0,00)$ y en los varones en la Mesomorfía, con un $(p=0,00)$. No existiendo en la Ectomorfía ( $p>0,05)$. No obstante, no se encontraron diferencias significativas al comparar los componentes de somatotipo entre las edades. A diferencia de los estudios de (Carter et al.; Rajan \& Rakshak Pal, 1997). En los que se aprecian modificaciones del somatotipo entre las diferentes edades.

En referencia a la información proporcionada por el IMC, se encontró un $31,8 \%$ de los varones y un 37,0\% de las damas presentaron obesidad y sobrepeso. Siendo inferiores a los valores encontrados en población escolar no-mapuche que tienen un $42,4 \%$ en varones y un $41,6 \%$ para las damas considerando los mismos parámetros (Martínez et al.).

En Chile como en todos los países del mundo ha habido procesos de transición nutricional, demográfica y epidemiológica Popkin (2001). El cambio nutricional está asociado a variables tales como el aumento de los ingresos y urbanización-modernización, ocio, trabajo, la influencia de los medios de comunicación de masas y el marketing de alimentos (Popkin, 1998, 2001; Rivera et al., 2002; Albala \& Vio, 2006). La etnia mapuche ha sufrido cambios en sus estilos de vida, producido por una migración de lo rural a lo urbano (Valenzuela). Esto posiblemente ha influido en nuevas conductas y patrones de actividad física, generando cambios en los hábitos alimenticios, sedentarismo y factores que influyen directamente en los índices de obesidad y sobrepeso encontrados en la población mapuche, los cuales no son similares a los encontrados por Espinoza et al. (2009) para la etnia Aymara la cual principalmente son sujetos enflaquecidos y normales en relación a su IMC.

El problema de la obesidad en Chile, afecta a la etnia mapuche, ya que producto de la migración de lo rural a lo urbano, se adapta a los cambios de la sociedad actual y desarrolla las conductas y estilo de vida propio de las áreas urbanas e industrializadas donde predomina un ambiente obesigénico.

AGRADECIMIENTOS. Los autores desean agradecer al Dr. Eric Díaz Bustos de la Universidad de la Frontera por su ayuda en el desarrollo de esta publicación.

MARTíNEZ, C.; SILVA, H.; COLLIPAL, E.; CARRASCO, V; RODRÍGUEZ, M.; VARGAS, R.; GATICA, P. \& SILVA, T. Somatotype and nutritional status from 10 to 14 years of age in a sample of Mapuche subjects in the IX Region, Temuco, Chile. Int. J. Morphol., 30(1):241-246, 2012.

SUMMARY: The Mapuche ethnic group has a wide distribution in the IX Region, the migration process of urban development itself, has made part of the Mapuche people move to the city of Temuco, modifying their lifestyles. The study of Body Mass Index (BMI) is the standard recommended by the WHO to know the nutritional status according to the technical standard nutritional norm in Chile. The somatotype is used to estimate body shape and composition. This study describes the nutritional situation and somatotype of a sample of Mapuche municipal schools of the city of Temuco. We evaluated 292 Mapuche adolescents of both sexes, 154 men (52.7\%) and 138 women (47.3\%), between 10 and 14 years of age. For somatotype anthropometric method was used described by Heath \& Carter while the assessment and classification of nutritional status according to technical standards nutrition. The men presented a mesomorphic somatotype (4.9/4.4). Women are endomesomórfico (5.4/4.3). The nutritional status of the sample has the following results in the case of males and $11.7 \%$ were obese and $20.1 \%$ were overweight. In checkers, $9.5 \%$ were obese and $27.5 \%$ were overweight. A comparison by gender differences were found in the endomorphy for women $(\mathrm{p}=0.00)$ and mesomorphy in boys, one $(\mathrm{p}=0.00)$. Not so in ectomorphy that did not differ. However, no differences were found when comparing the somatotype components between the ages of the sample. Obese-genic environment which emphasizes the modification of lifestyle, diet, sedentary effect is equally affecting the Mapuche population as the rest of the school population when compared with samples of students in municipal schools.

KEY WORDS: Mapuche subjects; Somatotype; Body mass index; Nutritional state. 


\section{REFERENCIAS BIBLIOGRÁFICAS}

Albala, C. \& Vio, F. Obesidad y pobreza: un desafío pendiente en Chile. In: Peña M, Bacallao J. La obesidad en la pobreza: un nuevo reto para la salud pública. Washington, Organización Panamericana de la Salud, 576:46-56, 2006.

Alegría, E.; Cordero, A.; Laclaustra, M.; Grima, A.; León, M.; Casanovas, J. A.; Luengo, E.; Del Río, A. \& Ferreira, I. Investigadores del registro MESYAS. Prevalencia del síndrome metabólico en población laboral española. El registro MESYAS. Rev. Esp. Cardiol., 58:797-806, 2005.

Almagià, F. A.; Toro, D. T.; Binvignat, G. O.; Cabrera, E. \& Marinao, A. Aproximación al perfil morfoestructural y dismorfismo sexual de jóvenes de ambos sexos de edad de 6 a 23 años de edad caracterizados por el somatotipo. Rev. Chil. Anat., 14:189-97, 1996.

Álvero, J. R, Cabañas, M. D.; Herrero de Lucas, A.; Martínez, L.; Moreno, C.; Porta, J.; Sillero, M. \& Sirvente, J. E. Protocolo de valoración de la composición corporal para el reconocimiento médico-deportivo. Documento de consenso del grupo español de cienantropometría de la federación española de medicina del deporte. Arch. Med. Deporte, 131:166-79, 2009.

Bibbins-Domingo, K.; Coxson, P.; Pletcher, M.J.; Lightwood, J. \& Goldman, L. Adolescent overweight and future adult coronary heart disease. N. Engl. J. Med., 357:2371-9, 2007.

Carter, J. \& Ackland, T. Sexual dimorphism in the physiques of World Championship divers. Sport. Sci., 16:317-29, 1998.

Carter, J. E. L. \& Heath, B. H. Somatotyping: development and applications. Cambridge, Cambridge University Press, 1990.

Carter, J. E. L.; Mirwald, R. L.; Heath-Roll, V. H. \& Bailey, D. A. Somatotypes of 7-to 16 year- old boys in Saskatchewan, Canada. Am. J. Hum. Biol., 9:257-72, 1997.

Cheng-Ye, J. P. \& Ohsawa, S. Changes in somatotype during growth in Chinese youth 7-18 years of Age. Am. J.Hum. Biol., 8:34759, 1996.

Del Sol, M. \& Henríquez, J. Evaluación de algunos índices antropométricos de un grupo de población mapuche. Bol. Mus. Araucano, 2:79-89, 1987.

Del Sol, M. \& Hunter, K. Evaluación postural de individuos mapuche de la zona costera de la IX Region de Chile. Int. J. Morphol., 22:339-42, 2004.

Del Sol, M. \& Olave, E. Índices claviculares en el grupo étnico mapuche. Rev. Chil. Anat., 14:183-8, 1996.

Del Sol, M. Índice Cefálico en un Grupo de Individuos Mapuches de la IX Región de Chile. Int. J. Morphol., 23:241-6, 2005.
Del Sol, M. Índices Faciales en Individuos Mapuches. Int. J. Morphol., 24:587-90, 2006.

Del Sol, M.; Vieira, M. C. \& Olave, E. Estudo morfométrico do esterno no grupo étnico Mapuche. Arq. Anat. Antropol., 41:2116, 1990 .

Díaz, E.; Saavedra, C.; Lira, M.; Bustos, E. \& Rivera, I. Evaluación de la condición física en adultos chilenos. Rev. Cs. Deporte del IND, 3:7-13, 2008.

Espinoza, O.; Vega, C.; Urrutia, A.; Moreno, A. \& Rodríguez, H. Patrones antropométricos y consumo máximo de oxigeno (VO2) entre niños escolares chilenos aimaras y no aimaras de 10 a 12 años, que viven en altura $(3.500 \mathrm{~ms})$ y la planicie $(500 \mathrm{~ms})$ Int . J. Morphol., 27:1313-8, 2009.

Ferrari, M. A.; Morazzani, F. \& Palacio-Tejedor, V. S. No es sólo comida comer en casa. En: Aquellos tehuelches de Patagonia Austral. Pinotti, L. (Comp). Buenos Aires, Colección Estudios de Salud y Población, Proyecto Editorial, 2004.

Henríquez, J.; Fuentes, R.; Sandoval, R \& Muñoz, A. Análisis de la estabilidad ortostática cráneocervical en adultos jóvenes mapuches. Int. J. Morphol., 21:149-53, 2003.

Instituto Nacional de Estadísticas (INE). Censo 2002, Síntesis de resultados. Santiago, INE, 2003.

ISAK (Sociedad internacional para el avance de la kinantropometría). Estándares Internacionales para la Evaluación Antropométrica. $1^{\mathrm{a}}$ ed. Sudáfrica, ISAK, 2001.

Katzmarzyk, P.; Malina, R.; Song, T. \& Bouchard, C. Physique, subcutaneous fat adipose tissue distribution, and risk factors in the Quebec family study. Int J. Obes. Relat. Metab. Disord., 23:476-84, 1999.

Katzmarzyk, P.; Malina, R.; Song, T. \& Bouchard, C. Somatotype and indicators of metabolic fitness in youth. Am. J. Hum. Biol., 10:341-50, 1998.

Magnusson, C.; Barón, J.; Persson, L.; Wolk, A.; Bergstrom, R.; Trichopoulos, D. \& Adami, H. O. Body size in different periods of life and breast cancer risk in post-menopausal women. Int. J. Cancer, 76:29-34, 1998.

Malina, R.; Bouchard, C. \& Bar-Or, O. Growth, Maturation and physical activity. 2. ed. Champaign, Human Kinetics, 2004.

Manolles, P.; Alcaraz, J.; Álvarez, J.; Jiménez, F.; Luengo, E.; Manus, B.; Naranjo, J.; Palacios, N.; Pérez, M. \& Villegas, J. La utilidad de la actividad física y los hábitos adecuados de nutrición como medio de prevención de la obesidad en niños y adolecentes. Arch. Med. Deporte, 127:333-53, 2008. 
MARTíNEZ, C.; SILVA, H.; COLLIPAL, E.; CARRASCO, V; RODRÍGUEZ, M.; VARGAS, R.; GATICA, P. \& SILVA, T. Somatotipo y estado nutricional de 10 a 14 años de edad en una muestra de mapuches de la IX Región, Temuco-Chile. Int. J. Morphol., 30(1):241-246, 2012.

Martínez, C.; Silva, H.; Collipal, E. \& Carrasco, V. Descripción del somatotipo e Imc en una muestra de adolecentes de colegios municipalizados de la ciudad de Temuco - Chile. Int. J. Morphol., 26:653-57, 2008.

Ministerio de Educación (MINEDUC), Unidad de Curriculum y Evaluación. Informe de resultados de Educación Física SIMCE $8^{\circ}$ básico, 2010. Disponible en: http://www.simce.cl/ fileadmin/Documentos_y_archivos_SIMCE/Informes_2010/ Informe_de_Resultados_Educacion_Fisica.pdf

Ministerio de Salud. Encuesta nacional de salud 2009-2010. Santiago, Ministerio de Salud, 2010.

Ministerio de Salud. La obesidad y el sobrepeso en Chile podrán afectar a más de nueve millones de personas en el año 2010. Santiago, Ministerio de Salud, 2003.

Ministerio de Salud. Norma Técnica de Evaluación Nutricional del niño de 6 a 18 años. Rev. Chil. Nutr., 31:128-37, 2004.

Norton, K. \& Olds, T. Antropométrica. 2nd ed. Sydney, Unsw Press, 2000. pp.148-69.

Organización Mundial de la Salud (OMS). Nota descriptiva: Obesidad y Sobrepeso, 2009. Disponible en: http://www.who.int/ topics/obesity/es/

Peña, M. \& Bacallao, J. Obesidad y pobreza un nuevo desafio de la salud pública. São Paulo, Roca. 2006.

Popkin, B. M. La transición de la nutrición y obesidad en el mundo en desarrollo. Diario de Nutrición, 871-3, 2001.

Popkin, B. M. La transición de la nutrición y sus implicaciones en países mal pagados. Nutrición de Salud pública, 5-21, 1998.

Rahmawati, N.; Budyharjo, S. \& Ashizawa, K. Somatotypes of young male athletes and non athletes students in yogyarta, Indonesia. Anthropological Science, 115:1-7, 2007.

Rajan, G. \& Rakshak Pal, S. Age differences in somatotypes of Garhwali males 17- 60 years of age. Am. J. Hum. Biol., 9:28590, 1997.

Rivera, J.; Barquera, S.; Campirano, F.; Campos, I.; Safdie, M. \& Tobar, V. Epidemiological and nutritional transition in Mexico: rapid increase of non- comunicable chronic diseases and obesit. Pub. Health Nutr., 5:123-8, 2002.

Saitoglu, M.; Ardicoglu, O.; Ozgocmen, S.; Kamanli, A. \& Kaya, A. Osteoporosis Risk factors and association with somatotypes in males. Arch. Med. Res., 38:746-51, 2007.

Sánchez-Muñoz, C.; Sanz, D. \& Zabala, M. Anthropometric characteristics, body composition and somatotype of elite junior tennis players. Br. J. Sports. Med., 41:793-9, 2007.
Silva, H.; Bruneau, J.; Reyno, P. \& Bucarey, S. Somatotipo e índice de masa corporal en una muestra de adolescentes de ambos sexos de la ciudad de Temuco, Chile. Int. J. Morphol., 21:30913, 2003.

Silva, H.; Collipal, E.; Martínez, C. \& Bruneau, J. Evaluación de los Componentes del Somatotipo e Índice de Masa Corporal en Escolares del Sector Precordillerano de la IX Región, Chile. Int. J. Morphol., 23:195-9, 2005.

Silva, H.; Collipal, E.; Martínez, C. \& Torres, I. Análisis del IMC y Somatotipo en una muestra de adolescentes con sobrepeso y obesidad en Temuco- Chile. Int. J. Morphol., 26:707-11, 2008.

Toro, T. \& Almagià, A. Aplicación de estándares antropométricos para la evaluación del crecimiento y estado nutricional en niñas y niños de 11-15 años de Valparaíso. Anal. Anat. Normal, 7:99-107, 1989.

Toro, T. \& Almagià, A. Mediciones biométricas, incluyendo grosor de pliegues cutáneos como indicadores de la composición corporal y estado nutricional. Estudio longitudinal en escolares de Valparaíso. Rev. Chil. Anat., 12:197-204, 1994.

Toro, T.; Arenas, G. \& Almagià, A. Caracterización somatotípica de jóvenes estudiantes de Valparaíso. Anal. Anat. Normal, 1:101-6, 1983.

Valenzuela, R. La población indígena de la Región Metropolitana. Santiago, CONADI, 1995.

Valkov, J.; Matev, T.; \& Hristov, I. Relation between somatype and some risk factors for ischemic heart disease. Folia Med. Plovdiv., 38:17-21, 1996.

Ventrella, R.; Semproli, S.; Jurimae, J.; Toselli, S.; Claessens, L.; Jurimae, T. \& Brasili, P. Somatotype in 6-11 year old Italian and Estonian schoolchildren. Homo, 59:383-96, 2008.

\section{Dirección para correspondencia \\ Prof. Cristian Martínez Salazar \\ Departamento de Educación Física \\ Universidad de la Frontera \\ Casilla 54 D, Temuco \\ CHILE}

Email: cmartin@ufro.cl

Recibido : 03-10-2011

Aceptado: 22-12-2011 